\title{
Towards Fixed Dosing of Tocilizumab in ICU-Admitted COVID-19 Patients: Results of an Observational Population Pharmacokinetic and Descriptive Pharmacodynamic Study
}

\author{
Dirk Jan A. R. Moes ${ }^{1,2}(1) \cdot$ David J. van Westerloo ${ }^{3}$. Sandra M. Arend ${ }^{4} \cdot$ Jesse J. Swen ${ }^{1,2}$. Annick de Vries ${ }^{5}$. \\ Henk-Jan Guchelaar $^{1,2}$. Simone A. Joosten ${ }^{4}$. Mark G. J. de Boer ${ }^{4}$. Teun van Gelder ${ }^{1,2}$. Judith van Paassen ${ }^{3}$
}

Accepted: 31 August 2021 / Published online: 11 October 2021

(c) The Author(s) 2021

\begin{abstract}
Background and Objective In the randomized controlled trial REMAP-CAP, it was shown that next to dexamethasone, the interleukin (IL)-6 receptor antagonist tocilizumab improves outcome, including survival in intensive care unit (ICU)admitted coronavirus disease 2019 (COVID)-19 patients. Therefore tocilizumab has been added to many COVID-19 treatment guidelines. Because obesity is a risk factor for the development of severe COVID-19, concerns have been raised about overtreatment, as well as undertreatment, through weight-based dosing of tocilizumab. The currently applied dose of $8 \mathrm{mg} /$ $\mathrm{kg}$ is based on the use of this drug for other indications, however it has not formally been investigated for COVID-19. In this study, the pharmacokinetics and pharmacodynamics of tocilizumab were investigated in ICU-admitted COVID-19 patients. Methods This was an open-label, single-centre, observational population pharmacokinetic and descriptive pharmacodynamic evaluation study. Enrolled patients, with polymerase chain reaction-confirmed COVID-19 were admitted to the ICU for mechanical ventilation or high flow nasal canula oxygen support. All patients were 18 years of age or older and received intravenous tocilizumab $8 \mathrm{mg} / \mathrm{kg}$ (maximum $800 \mathrm{mg}$ ) within $24 \mathrm{~h}$ after admission to the ICU and received dexamethasone $6 \mathrm{mg}$ daily as concomitant therapy. For evaluation of the pharmacokinetics and pharmacodynamics of tocilizumab, all time points from day 0 to 20 days after dose administration were eligible for collection. A nonlinear mixed-effects model was developed to characterize the population pharmacokinetic parameters of tocilizumab in ICU-admitted COVID-19 patients. Covariate analysis was performed to identify potential covariates for dose individualization. For the development of alternative dosing schedules, Monte Carlo simulations using the final model were performed.

Results Overall, 29 patients were enrolled between 15 December 2020 and 15 March 2021. A total of 139 tocilizumab plasma samples were obtained covering the pharmacokinetic curve of day 0 to day 20 after tocilizumab initiation. A population pharmacokinetic model with parallel linear and nonlinear clearance (CL) was developed and validated. Average CL was estimated to be $0.725 \mathrm{~L} /$ day, average volume of distribution $\left(V_{\mathrm{d}}\right)$ was $4.34 \mathrm{~L}$, maximum elimination rate $\left(V_{\max }\right)$ was $4.19 \mu \mathrm{g} /$ day, and concentration at which the elimination pathway is half saturated $\left(K_{\mathrm{m}}\right)$ was $0.22 \mu \mathrm{g} / \mathrm{mL}$. Interindividual variability was identified for CL $(18.9 \%)$ and $V_{\mathrm{d}}(21 \%)$. Average area under the concentration versus time curve from time zero to infinity of the first dose $\left(\mathrm{AUC}_{\text {inf } 1 \text { st DOSE }}\right)$ was $938[ \pm 190] \mu \mathrm{g} / \mathrm{mL} *$ days. All patients had tocilizumab exposure above $1 \mu \mathrm{g} / \mathrm{mL}$ for at least 15 days. Bodyweight-based dosing increases variability in exposure compared with fixed dosing.

Conclusions This study provides evidence to support a fixed dose of tocilizumab $600 \mathrm{mg}$ in COVID-19 patients. Fixed dosing is a safe, logistically attractive, and drug expenses saving alternative compared with the current $8 \mathrm{mg} / \mathrm{kg}$ recommendation.
\end{abstract}

\section{Introduction}

The severe acute respiratory syndrome coronavirus 2 (SARS-Cov-2) pandemic has caused over 212,000,000 coronavirus cases and over 4,400,000 deaths worldwide [1]. The inflammatory storm associated with SARS-CoV-2 infection damages the respiratory tract and causes high

Extended author information available on the last page of the article morbidity and mortality [2]. It was recently shown in the REMAP-CAP open-label, randomized controlled trial that next to dexamethasone, treatment with the interleukin (IL)-6 receptor antagonists tocilizumab and sarilumab significantly improved outcomes, including survival in intensive care unit (ICU)-admitted coronavirus disease 2019 (COVID-19) patients with acute respiratory distress syndrome (ARDS) [3-5]. Tocilizumab is the first marketed IL-6-blocking 


\section{Key Points}

In the randomized controlled trial REMAP-CAP, the interleukin (IL)-6 receptor antagonist tocilizumab was shown to improve outcome, including survival in intensive care unit (ICU)-admitted coronavirus disease 2019 (COVID-19) patients. Therefore, tocilizumab has been added to many COVID-19 treatment guidelines. The current proposed dose of tocilizumab $8 \mathrm{mg} / \mathrm{kg}$ ( 800 mg maximum) is based on the standard loading dose of rheumatoid arthritis, however evidence is lacking that this is also the optimal dose for COVID-19 acute respiratory distress syndrome.

Because obesity is a risk factor for the development of severe COVID-19, concerns have been raised about overtreatment, as well as undertreatment, through weight-based dosing of tocilizumab. Furthermore, pharmacokinetic and pharmacodynamic parameters of medications are often found to be different in severely ill patients when compared with mild or moderately ill patients. However, the effects of different dosing schedules were only investigated to a very limited extent in nonrandomized observational studies. Hence, evaluation of the pharmacokinetics and pharmacodynamics of tocilizumab in severely ill COVID-19 patients is warranted.

This study provides valuable information about the population pharmacokinetics and descriptive pharmacodynamics of tocilizumab in dexamethasone co-treated ICU-admitted COVID-19 patients. This research shows that there is more rationale for a fixed dosing recommendation than for the current $8 \mathrm{mg} / \mathrm{kg}$ dosing recommendation in the investigated patient population. Due to the ongoing pandemic, shortages of tocilizumab and other IL-6 receptor antagonists may be anticipated. A fixed tocilizumab dose regimen of $600 \mathrm{mg}$ has many practical and safety advantages, e.g. it will reduce dosing errors and avoid unnecessary wastage of medication and is a logistically attractive alternative.

humanized antibody targeting IL-6 receptors, and has proved its safety and effectiveness in therapy for rheumatoid arthritis (RA) [6, 7]. The current proposed dose of tocilizumab 8 $\mathrm{mg} / \mathrm{kg}$ (800 mg maximum) is based on the standard loading dose of RA, however evidence is lacking that this is also the optimal dose for COVID-19 ARDS [8]. Tocilizumab is currently also registered for cytokine release syndrome (CRS) that can develop after chimeric antigen receptor $\mathrm{T}$ lymphocyte (CAR-T) cell therapy. Interestingly, the US FDA registration report consists of limited data of the CRS population and was further based on modelling and simulation studies based on data from RA patients. The FDA review committee concluded that as the maximum tolerated dose was not reached in previous trials for tocilizumab, the 'safety threshold' from previous clinical experience was used to determine the acceptable dose regimen and that the optimal dose and schedule are not well-established [9]. Underdosing of tocilizumab might lead to suboptimal suppression of hyperinflammation, and overdosing might result in increased risk for secondary antimicrobial infections and unnecessary drug expenses. For optimization of the dose regimen of tocilizumab, population pharmacokinetic and pharmacodynamic studies are essential. The pharmacokinetics and pharmacodynamics of tocilizumab in severe COVID-19 patients is still not well understood and no published data of tocilizumab pharmacokinetics and pharmacodynamics in this population are available. The pharmacokinetics of tocilizumab might be different in COVID-19 patients compared with RA and CRS patients. In addition, a wide range of interindividual variability (IIV) in the estimated pharmacokinetic parameters of tocilizumab has been demonstrated in RA, and this variability might even be higher in ICU-admitted COVID19 patients [8]. The primary objective of this study was to characterize the population pharmacokinetics and descriptive pharmacodynamics of tocilizumab in ICU-admitted COVID-19 patients, while the secondary objective was to identify potential covariates that influence the pharmacokinetics of tocilizumab and to develop an alternative dosing recommendation for tocilizumab ICU-admitted COVID-19 patients.

\section{Methods}

\subsection{Study Design and Participants}

This was an open-label, observational pharmacokinetic and pharmacodynamic evaluation study. Critically ill patients aged 18 years of age or older with polymerase chain reaction (PCR)-confirmed COVID-19 who were admitted to the ICU and receiving respiratory organ support were classified as having a severe disease state and were eligible for tocilizumab administration according to the local treatment protocol. The local drug treatment protocol consisted of dexamethasone $6 \mathrm{mg}$ once daily starting from hospital admission for a maximum of 10 days, no antiviral therapy and a single dose of tocilizumab administration $(8 \mathrm{mg} / \mathrm{kg}$ ) within $24 \mathrm{~h}$ of ICU admission, which was the same as the nationwide guideline [10]. Respiratory organ support was defined as either high flow oxygen support (flow rate $>40 \mathrm{~L}$, oxygen fraction $>0.4$ ), or invasive or noninvasive mechanical ventilation. Tocilizumab $8 \mathrm{mg} / \mathrm{kg}$ (800 mg maximum) was administered within $24 \mathrm{~h}$ after starting organ support in the ICU. All patients were admitted at the Leiden University Medical 
Center, Leiden, The Netherlands, between 15 December 2020 and 15 March 2021. Recruitment started on 15 December 2020 and ended on 15 February 2021. All patients were followed-up until 25 days after tocilizumab administration unless they were discharged or had died during the follow-up period. Data and sample collection was performed between 15 December 2020 and 1 April 2021. EDTA plasma samples were obtained from day 0 to day 20 after tocilizumab administration. Furthermore, demographic factors, including sex, age, bodyweight, height, body mass index (BMI), body surface area (BSA) and routinely collected clinical chemistry laboratory values (serum creatinine, estimated glomerular filtration ratio according to the Chronic Kidney Disease Epidemiology Collaboration [CKD-EPI; eGFR], urea, total bilirubin, albumin, cytokine reactive protein [CRP], aspartate aminotransferase [ASAT], alanine aminotransferase [ALAT], $\gamma$-glutamyltransferase [GGT], ferritin, D-dimer) were obtained from electronic healthcare records. The study was conducted in compliance with the Declaration of Helsinki and was approved by the COVID-19 Scientific and Ethics Committee of the Leiden University Medical Center with number coco 2020-033. Furthermore, a waiver for the requirement for informed consent was granted.

\subsection{Sampling and Bioanalysis}

For evaluation of the pharmacokinetics of tocilizumab, all time points from day 0 to 20 days after dose administration were eligible for collection. Daily sampled leftovers from samples of the haematology tubes (K2-EDTA whole blood) were used to obtain plasma for free tocilizumab concentration determination. Plasma was obtained after centrifugation and stored at $-20^{\circ} \mathrm{C}$ until analysis. Tocilizumab concentrations were determined in the Sanquin Biologics Lab, Amsterdam, The Netherlands. Tocilizumab concentrations were determined with a validated enzymelinked immunosorbent assay (ELISA) method using rabbit anti-tocilizumab antibodies to capture tocilizumab, and rabbit anti-tocilizumab $\mathrm{F}\left(\mathrm{ab}^{\prime}\right) 2$ fragments for detection as described earlier [11, 12]. Maxisorp ELISA plates were then coated overnight at room temperature with $0.125 \mu \mathrm{g} / \mathrm{mL}$ rabbit anti-tocilizumab in phosphate-buffered saline (PBS). The specific rabbit anti-idiotype antibodies were produced analogously, as described for natalizumab [11, 12]. Absorption was measured at $450 \mathrm{~nm}$ relative to a titration curve of tocilizumab in each plate. The lower limit of quantification (LLOQ) in plasma was $0.2 \mu \mathrm{g} / \mathrm{mL}$, and the overall precision and accuracy were $8 \%$ and $93 \%$, respectively. Samples exceeding the upper limit of quantification of $250 \mu \mathrm{g} / \mathrm{mL}$ were diluted. Plasma soluble interleukin-6 receptor (sIL-6R) was determined by ELISA in duplicate using the commercial Quantikine Human IL-6sR kit (Bio-Techne Ltd, Abington, UK) as described earlier [6].

\subsection{Population Pharmacokinetic Modelling}

The sample size determination was based on the sample size of the registration study of the CRS indication for tocilizumab approved by the FDA [9]. A nonlinear mixed-effects model was developed to characterize the pharmacokinetic parameters of tocilizumab in ICU-admitted COVID-19 patients using the first-order conditional estimation with interaction approach. One- and two-compartmental models with and without (parallel) nonlinear pharmacokinetics were evaluated. During model development, candidate models were evaluated for their decrease in objective function value (OFV) calculated as the $-2 \log$ likelihood. A decrease in OFV of $\geq 6.63$ was considered significant (Chi-square, 1 degree of freedom $[d f], p<0.01$ ). In addition, basic goodness-of-fit (GOF) plots, in which the observed concentration is plotted against the individual- and population-predicted concentrations, and the conditional-weighted residual errors are plotted against time- and population-predicted concentrations and normalized prediction distribution error (NPDE) plots, were assessed. Furthermore, parameter precision, shrinkage and inter individual variability (IIV) were taken into account during the modelling process. A posterior power calculation was performed using the stochastic simulation and estimation tool Perl-speaks-Nonlinear Mixed Effects Modelling (NONMEM)(PsN; v.5.0.0) to calculate the power to identify the covariate effect of bodyweight as an exponent on tocilizumab clearance $(\mathrm{CL})$.

\subsubsection{Covariate Analysis}

Biological plausibility of a potential covariate was a selection criteria for the statistical assessment of covariates. Baseline demographic covariates (sex, age, bodyweight, BMI, BSA, and height) and laboratory covariates (creatinine, eGFR, urea, albumin, lactate dehydrogenase [LDH], ASAT, ALAT, GGT, total bilirubin, albumin, CRP) were initially included in the visual covariate analysis. Elimination of tocilizumab is assumed to be a combination of the linear elimination through nonspecific binding and a targetmediated elimination related to tocilizumab binding to both the sIL-6R and membrane-expressed IL-6 receptor (mIL6R) and subsequent elimination of the drug-target complex [13]. Demographic variables such as bodyweight and BSA are the most frequent and clinically relevant covariates found in studies on monoclonal antibody pharmacokinetics. Next to disease variables such as CRP, albumin has been shown to influence monoclonal antibody pharmacokinetics [14]. Bodyweight, albumin and CRP specifically have previously been associated with tocilizumab pharmacokinetics $[8,15]$. The influence of time-varying covariates could be assessed for CRP, creatinine, eGFR, urea, albumin, LDH, ASAT, ALAT, GGT, total bilirubin and albumin. The covariates 
LDH, ASAT, ALAT and GGT were only assessed since they could potentially influence dexamethasone pharmacokinetics. Glucocorticoids are known to diminish the production of cytokines, including IL-6. A subsequent reduction of free IL-6 receptor expression may potentially influence tocilizumab pharmacokinetics [16]. Covariates were subsequently entered in the univariate statistical analysis (Chi-square, 1 $d f, p<0.05$ ) and subsequently, but only if applicable, in the cumulative forward inclusion/backward elimination procedure. Continuous covariates were tested as linear, piecewise linear, power, and exponential functions, and categorical covariates were tested as linear function. Specifically, bodyweight was also tested as an allometric function. A covariate was only retained if it led to a significantly improved fit (Chi-square, $1 d f, p<0.01)$. Graphical displays based on the agreement between the observed and predicted drug concentrations and the uniformity of the distribution of the residuals were also considered for covariate inclusion.

\subsubsection{Model Evaluation, Model Validation and Software}

The final model was evaluated by means of a predictioncorrected visual predictive check (pcVPC) based on 500 Monte-Carlo simulations. In addition, the precision of the parameter estimates was further assessed by means of a nonparametric bootstrap with resampling the dataset $(n=1000$ times). The population pharmacokinetic modelling was carried out using NONMEM v.7.4.4 (Icon Development Solutions, Ellicott City, MD, USA) and Perl Speaks NONMEM (v.5.0.0) [17-19]. Pirana was used for run interpretation (v. 2.9.8) [20], and R statistics (v. 3.4.4) was used for exploratory graphical analysis and for evaluation of the GOF, NPDE (NPDE package 3.0) [21] and pcVPC [22].

\subsection{Outcomes}

Primary endpoints were the pharmacokinetic and pharmacodynamic (CRP and sIL-6R) parameters of tocilizumab in ICU-admitted COVID-19 patients. Next to the population pharmacokinetic parameters of $\mathrm{CL}$, volume of distribution $\left(V_{\mathrm{d}}\right)$, maximum elimination rate $\left(V_{\max }\right)$, concentration at which the elimination pathway is half saturated $\left(K_{\mathrm{m}}\right)$, area under the concentration versus time curve from zero to infinity of the first dose $\left(\mathrm{AUC}_{0 \text {-inf 1st DOSE }}\right)$ and average concentration $\left(C_{\text {avg }}\right)$ were also calculated. Secondary endpoints were identification of potential covariates and the development of alternative dosing schedules. An exploratory endpoint was the difference in these pharmacokinetic parameters in subgroups such as survivors and nonsurvivors, which was assessed using an independent samples $t$-test. For the development of alternative dosing schedules, Monte Carlo simulations in NONMEM using the final model were performed to assess whether bodyweight dosing or fixed dosing was more appropriate to reduce variability in tocilizumab exposure. The predicted concentration time curves were simulated 1000 times for patients receiving $8 \mathrm{mg} / \mathrm{kg}$ for a bodyweight of $60,70,80,90$ and $100 \mathrm{~kg}$, which represents the bodyweight range in the current study population compared with a fixed dose of $600 \mathrm{mg}$ for all bodyweight categories. Furthermore, predicted concentration time curves for fixed doses of $800,600,400$ and $200 \mathrm{mg}$ and more personalized alternative regimens were simulated.

\section{Results}

\subsection{Patients and Samples}

Twenty nine ICU-admitted COVID-19 patients treated with dexamethasone $6 \mathrm{mg}$ daily as well as tocilizumab $8 \mathrm{mg} / \mathrm{kg}$ were studied for up to 25 days after tocilizumab administration. Patients had a mean age of 64 years (range 45-80), the majority of patients were male $(72.4 \%)$, and mean total bodyweight was $96.4 \mathrm{~kg}$ (range 58-130). All patients received concomitant anti-inflammatory treatment with dexamethasone $6 \mathrm{mg}$ once daily for a maximum of 10 days during their hospital admission; 24\% received methylprednisolone in addition as rescue therapy when the initial course of dexamethasone and tocilizumab did not lead to clinical recovery after 10 days. Median time from COVID-19 first symptoms until tocilizumab administration was 11.7 days (range 1.6-24.6). All patients received tocilizumab $8 \mathrm{mg} / \mathrm{kg}$, with a maximum of $800 \mathrm{mg}$. Only one patient received a double dose, accidentally, and another patient received the total dose of $8 \mathrm{mg} / \mathrm{kg}$ in two steps (within $12 \mathrm{~h}$ ) because tocilizumab was temporarily out of stock. Detailed baseline characteristics of the studied population can be found in Table 1. A total of 139 tocilizumab concentration-time points were obtained, ranging from day 1 until day 20 after dose administration, covering the entire pharmacokinetic curve. The mean and median number of samples per patient was 4.8 and 5 samples, respectively, with a range of 1-11 samples. All obtained data were used for the population pharmacokinetic analysis. In 80 of the collected samples evenly divided over all patients, sIL-6R measurements could be performed.

\subsection{Model Development and Validation}

During the model building process, summarized in Table 2, one- and two-compartment models with and without parallel linear and nonlinear elimination were evaluated. A twocompartment model that was identified earlier in RA and CRS, with parallel first-order (linear) and Michaelis-Menten (nonlinear) elimination kinetics, was also explored [8, 9, 23]. The tocilizumab plasma concentration data of the current study were ultimately best described by a one-compartment 
disposition model with parallel first-order (linear) and Michaelis-Menten (nonlinear) elimination kinetics (Fig. 1). An overview of the obtained concentration data and the individual model fit is presented on a semi-log scale and different colour gradient per individual (Fig. 2). The diagnostic plots for the basic and final population pharmacokinetic model indicated that the observed and predicted data were in good agreement. Average CL was estimated to be $0.725 \mathrm{~L} /$ day, average $V_{\mathrm{d}}$ was $4.34 \mathrm{~L}, V_{\max }$ was $4.19 \mathrm{mg} /$ day and $K_{\mathrm{m}}$ was $0.22 \mathrm{mg} / \mathrm{L}$. IIV was identified for CL $(18.9 \%)$ and $V_{\mathrm{d}}(21 \%)$. Residual variability was characterized by a combined error model with an additive error of $0.139 \mu \mathrm{g} / \mathrm{mL}$ and a proportional error of $17.1 \%$ (expressed as the coefficient of variation). A complete overview of the pharmacokinetic parameter estimates from the final population pharmacokinetic model, including the statistical distributions of the estimates obtained from the bootstrap, is shown in Table 3. The median values of the parameters estimated from the bootstrap analysis were in good agreement with the point estimates, and the $95 \%$ confidence intervals (CIs) were reasonably narrow, demonstrating acceptable precision. Furthermore, the model parameters had adequately low levels of $\eta$-shrinkage for CL (8\%) and $\mathrm{V}_{\mathrm{d}}(11 \%)$. pcVPC (on normal and semi-log scales) demonstrated good predictive performance of the final population pharmacokinetic model (electronic supplementary material [ESM] Figs. S1 and S2). Furthermore, all GOF plots showed good model performance, both on normal and loglog scales (ESM Fig. S3). An additional NPDE evaluation (ESM Fig. S4) showed adequate results and the NONMEM final model code can be found in ESM 1.

\subsection{Covariate Analysis}

The baseline demographic covariates (sex, bodyweight, age, BMI, BSA, height) and laboratory covariates (creatinine, eGFR, urea, albumin, LDH, ASAT, ALAT, GGT, total bilirubin, albumin, CRP) did not show any relationship between $\mathrm{CL}$ or $V_{\mathrm{d}}$ after visual and descriptive analysis. Bodyweight was tested as a first covariate in the univariate analysis. During the univariate covariate analysis no statistically significant covariates were identified (all $p>0.05$ ).

Therefore, also after the multivariate covariate statistical analysis, no statistically significant covariates were identified. The most important and biological plausible covariate deviations of the individual's parameters from the typical mean (ETA) plots for CL and $V_{\mathrm{d}}$ can be found in ESM Fig. S5.

\subsection{Pharmacokinetics and Descriptive Pharmacodynamics}

The average $\mathrm{AUC}_{0 \text {-inf 1st DOSE }}$ was $938[ \pm 190] \mu \mathrm{g} / \mathrm{mL} *$ days. In RA patients, Nishimoto et al. showed that tocilizumab normalized the CRP level as long as the free tocilizumab, which is capable of binding IL-6R and inhibiting IL-6 actions, remained above $1 \mu \mathrm{g} / \mathrm{mL}$ in serum. All patients in our cohort had at least 15 days of tocilizumab exposure above $1 \mu \mathrm{g} / \mathrm{mL}$ [6]. On average, patients with lower bodyweight had lower exposure than patients with higher bodyweight. Figure 3 displays the individual predicted concentration-time curves, measured tocilizumab concentrations, CRP value pharmacodynamics and the threshold of $1 \mu \mathrm{g} / \mathrm{mL}$ of all included patients on a semi-log scale. Most variability in exposure is present in the first days after tocilizumab administration. In all patients, CRP declined rapidly after tocilizumab administration and remained low for at least 10 days. In a minority of patients ( 8 of 29), a clear new increase in CRP levels was observed 10 days after dose administration despite detectable tocilizumab concentrations. Four of these patients were diagnosed with a secondary infection as described in the electronic healthcare record. As shown in Fig. 2, tocilizumab is detectable in all patients for at least 15 days after dose administration and even longer for the majority of patients. Individual plots per patient on a semi-log scale are shown in ESM 2. Figure 4 shows the individual predicted concentration-time curves, measured tocilizumab concentration, CRP value pharmacodynamics and the threshold of $1 \mathrm{ug} / \mathrm{mL}$ of patients with (Fig. 4a) and without (Fig. 4a) a clear new increase in CRP levels separately. The group of patients with a relapse of CRP was more thoroughly compared with the non-CRP relapse group with regard to tocilizumab exposure and baseline CRP. The results are presented in ESM Table S1. No statistically significant differences in pharmacokinetic parameters were identified (all $p>0.05$ ). Only a trend to higher baseline CRP was present in the CRP relapse group. A total of 11 patients died during or after ICU admission, and the majority (64\%) of these patients died within the first 25 days after tocilizumab administration. No statistically significant differences in tocilizumab pharmacokinetic parameters were found between survivors and nonsurvivors, as presented in ESM Table S2 (all $p>0.05$ ). Figure 5 shows the individual predicted concentration-time curves, measured tocilizumab concentration, sIL-6R value pharmacodynamics and the threshold of $1 \mu \mathrm{g} / \mathrm{mL}$ of all included patients on a semi$\log$ scale. The sIL-6R concentrations increased clearly after tocilizumab administration and seemed to slowly decline in some patients 17 days after administration. Individual plots per patient on a semi-log scale are shown in ESM 3. Due to target-mediated drug disposition, the nonlinear part of the $\mathrm{CL}$ of tocilizumab is dominant in low concentration ranges and the linear part is dominant in high concentration ranges [8]. The nonlinear elimination pathway of tocilizumab represents a target-mediated CL process due to binding to IL-6R. Based on the final model parameters, the threshold of when the nonlinear CL exceeds the linear CL in this 
Table 1 Baseline and clinical characteristics

\begin{tabular}{|c|c|c|}
\hline Baseline characteristics & Mean & Range \\
\hline Male $(\%)$ & 72.4 & \\
\hline Age (years) & 64 & $45-80$ \\
\hline Bodyweight (kg) & 96.4 & $58.0-130.0$ \\
\hline Height (cm) & 177 & $165-192$ \\
\hline BMI & 30.9 & $20.1-46.1$ \\
\hline BSA & 2.2 & $1.7-2.5$ \\
\hline Smoking & 4 & \\
\hline Ureum $(\mathrm{mmol} / \mathrm{L})$ & 10 & $3.8-19.5$ \\
\hline Creatinine (umol/L) & 87 & $48-167$ \\
\hline eGFR $\left(\mathrm{mL} / \mathrm{min} / 1.73 \mathrm{~m}^{2}\right)$ & 78 & $37-100$ \\
\hline Albumin $(\mathrm{g} / \mathrm{L})$ & 32 & $23-37$ \\
\hline LDH (U/L) & 565 & $197-1826$ \\
\hline $\mathrm{CRP}(\mathrm{mg} / \mathrm{L})$ & 146 & $13.4-309$ \\
\hline ASAT (U/L) & 59 & 14-314 \\
\hline ALAT (U/L) & 59 & 10-209 \\
\hline GGT (U/L) & 125 & $16-1194$ \\
\hline Total bilirubin (umol/L) & 12 & $3-42$ \\
\hline Ferritin $(\mu \mathrm{g} / \mathrm{L})$ & 1704 & $407-4441$ \\
\hline D-Dimer (ng/mL) & 8737 & $333-83,500$ \\
\hline Temperature $\left({ }^{\circ} \mathrm{C}\right)$ & 36.6 & $35.3-39.2$ \\
\hline CT scan long infiltration (\%) & 53 & $15-85$ \\
\hline CT score severeness (maximum 25) & 17.2 & $8-24$ \\
\hline Tocilizumab dose administered (mg) & 781 & $472-1552$ \\
\hline Tocilizumab initiation (days after the first symptoms) & 11.7 & $1.6-24.6$ \\
\hline Tocilizumab initiation (days after LUMC ICU admission) & 1.7 & $0.1-11.7$ \\
\hline Follow-up (days) & 16.1 & $0.9-25.5$ \\
\hline Mechanical ventilation (\%) & 82.8 & \\
\hline Ventilation in the abdominal position (\%) & 62.1 & \\
\hline SOFA score & 7.5 & $3-12$ \\
\hline APACHE IV score & 71.6 & $41-123$ \\
\hline SAPS III score & 43.9 & $37-52$ \\
\hline \multicolumn{3}{|l|}{ Comorbidities (\%) } \\
\hline Diabetes & 6.9 & \\
\hline Hypertension & 41.4 & \\
\hline Cardiovascular disease & 31.0 & \\
\hline Chronic renal insufficiency & 6.9 & \\
\hline Cancer & 13.8 & \\
\hline COPD & 13.8 & \\
\hline Anti-inflammatory comedication & & - \\
\hline Dexamethasone & 100 & - \\
\hline Methylprednisolone & 24.1 & - \\
\hline Hydrocortisone & 3.4 & - \\
\hline
\end{tabular}

$B M I$ body mass index, $B S A$ body surface area, $e G F R$ estimated glomerular filtration ratio, $L D H$ lactate dehydrogenase, CRP cytokine reactive protein, ASAT aspartate aminotransferase, ALAT alanine aminotransferase, $G G T \gamma$-glutamyltransferase, $C T$ computed tomography, LUMC Leiden University Medical Center, ICU intensive care unit, SOFA Sequential Organ Failure Assessment, APACHE Acute Physiology and Chronic Health Evaluation, SAPS III Simplified Acute Physiology Score III, COPD chronic obstructive pulmonary disease 
Table 2 Model building process

\begin{tabular}{|c|c|c|c|c|c|c|c|}
\hline $\begin{array}{l}\text { Model } \\
\text { num- } \\
\text { ber }\end{array}$ & Description & Model & $\begin{array}{l}\text { Com- } \\
\text { pared } \\
\text { against }\end{array}$ & OFV & $\Delta \mathrm{OFV}$ & AIC & Comment \\
\hline 1 & $\begin{array}{l}\text { One-compartment with linear } \\
\text { elimination }\end{array}$ & $\begin{array}{l}\mathrm{CL}=\Theta 1 \times \operatorname{EXP}(\text { IIV) } \\
V_{d}=\Theta 2 \times \operatorname{EXP}(\text { IIV })\end{array}$ & & 821.532 & & 835.532 & \\
\hline 2 & $\begin{array}{l}\text { One-compartment with parallel } \\
\text { linear and nonlinear elimina- } \\
\text { tion }\end{array}$ & $\begin{array}{l}\mathrm{CL}=\Theta 1 \times \mathrm{EXP}(\mathrm{IIV}) \\
V_{\mathrm{d}}=\Theta 2 \times \mathrm{EXP}(\mathrm{IIV}) \\
\mathrm{K}_{\mathrm{m}}=\Theta 3 \\
V_{\max }=\Theta 4\end{array}$ & 1 & 792.078 & -29.452 & 810.08 & $\begin{array}{l}\text { No IIV identifiable for } K_{\mathrm{m}} \text { and } \\
V_{\max }\end{array}$ \\
\hline 3 & $\begin{array}{l}\text { Two-compartment with parallel } \\
\text { linear and nonlinear elimina- } \\
\text { tion }\end{array}$ & $\begin{array}{l}\mathrm{CL}=\Theta 1 \times \mathrm{EXP}(\mathrm{IIV}) \\
V_{c}=\Theta 2 \times \mathrm{EXP}(\mathrm{IIV}) \\
V_{p}=\Theta 3 \\
Q=\Theta 4 \\
\mathrm{~K}_{\mathrm{m}}=\Theta 5 \\
V_{\max }=\Theta 6\end{array}$ & 2 & 785.761 & -6.317 & 807.761 & $\begin{array}{l}\text { Very high RSEs }(>1000 \%) \text { and } \\
\text { unstable parameter estimates. } \\
\text { No IIV identifiable for } Q, K_{\mathrm{m}} \\
\text { and } V_{\max }\end{array}$ \\
\hline 4 & $\begin{array}{l}\text { One-compartment with parallel } \\
\text { linear and nonlinear elimina- } \\
\text { tion }\end{array}$ & $\begin{array}{l}\mathrm{CL}=\Theta 1 \times \mathrm{EXP}(\mathrm{IIV}) \\
V_{\mathrm{d}}=\Theta 2 \times \operatorname{EXP}(\mathrm{IIV}) \\
\mathrm{K}_{\mathrm{m}}=\Theta 3 \\
V_{\max }=\Theta 4\end{array}$ & & 792.078 & & 810.08 & $\begin{array}{l}\text { Base model for covariate analysis, } \\
\text { no significant covariates }\end{array}$ \\
\hline 5 & $\begin{array}{l}\text { One-compartment with parallel } \\
\text { linear and nonlinear elimina- } \\
\text { tion }\end{array}$ & $\begin{array}{l}C L=\Theta 1 \times \operatorname{EXP}(\text { IIV }) \\
V_{d}=\Theta 2 \times \operatorname{EXP}(\text { IIV }) \\
K_{m}=\Theta 3 \\
V_{\max }=\Theta 4\end{array}$ & & 792.078 & & 810.08 & Final model \\
\hline
\end{tabular}

$O F V$ objective function value derived from NONMEM, $C L$ clearance, $V_{d}$ volume of distribution, $V_{c}$ volume of distribution in the central compartment, $V p$ volume of distribution in the peripheral compartment, $Q$ intercompartmental clearance, IIV interindividual variability, AIC Akaike Information Criterion, $K_{m}$ concentration at which the elimination pathway is half saturated, RSEs relative standard errors

patient population was estimated to be $5 \mu \mathrm{g} / \mathrm{mL}$, as shown in Fig. 6. Exposures above this threshold will lead to more pronounced saturation of the nonlinear CL.

\subsection{Bodyweight Dosing Versus Fixed Dosing}

To estimate the bodyweight exponent, in order to compare the exponent with the range of $0-1$ as proposed by Wang et al., the bodyweight was also tested as a covariate to the model as shown in Eq. 1:

$\mathrm{CL}=\mathrm{CL} \times\left(\frac{\mathrm{BW}}{\text { median BW}}\right)^{\text {exp }}$

The exponent was estimated to be as low as 0.002 , which is almost 0 , meaning no effect at all on CL. Furthermore, the inclusion did not lead to a statistically significant improvement of the model, nor did it reduce the IIV in CL. As explained by Wang et al., an exponent of as high as 1 for a power function covariate model would describe a proportional change of a pharmacokinetic parameter with change in the covariate. The exponent of the covariate model for body size effect on the CL of a linear elimination pathway ranges from 0 (body size is not a covariate) to 1.2. Which dosing approach is better depends on how steep the relationship is between CL and body size, that is, the value of the exponent. Fixed dosing provides less variability in exposure when exp
$<0.5$, while this is true for bodyweight-based dosing when $\exp >0.5$ [24]. Due to the absence of bodyweight-dependent $\mathrm{CL}$, an increasing $\mathrm{AUC}_{0 \text {-inf 1st DOSE }}$ can be observed when bodyweight increases in the current dataset, as shown in ESM Fig. S6.

The final model, which in this case is the same as the base model, was used to simulate the distribution of the concentration-time profiles of tocilizumab in patients weighing $60-100 \mathrm{~kg}$, which is representative of the investigated study population. In Fig. 7, the variability in concentration versus time curves is presented for 1000 simulated patients weighing between 60 and $100 \mathrm{~kg}$ receiving a tocilizumab $8 \mathrm{mg} / \mathrm{kg}$ dose, versus all patients receiving a $600 \mathrm{mg}$ fixed dose on a semi-log scale. The normal scale plot is presented in ESM Fig. S7. Furthermore, the 1 and $5 \mu \mathrm{g} / \mathrm{mL}$ receptor saturation thresholds are shown. Figure 7 and ESM Fig. S7 (normal scale) show that bodyweight-based dosing increases variability in exposure (especially peak concentrations) compared with fixed dosing. Moreover, with fixed dosing, tocilizumab is above the 1 and $5 \mu \mathrm{g} / \mathrm{mL}$ threshold for a similar duration as bodyweight-based dosing, with a minimum of approximately 15 days. Figure 8 and ESM Fig. S8 (normal scale) show simulations of 1000 patients for the duration of tocilizumab exposure above these two thresholds, when using different alternative fixed doses of 800, 600, 400, 200 $\mathrm{mg}$. Even with the lowest fixed dose of $200 \mathrm{mg}$, tocilizumab 


\section{Population Pharmacokinetic Model}

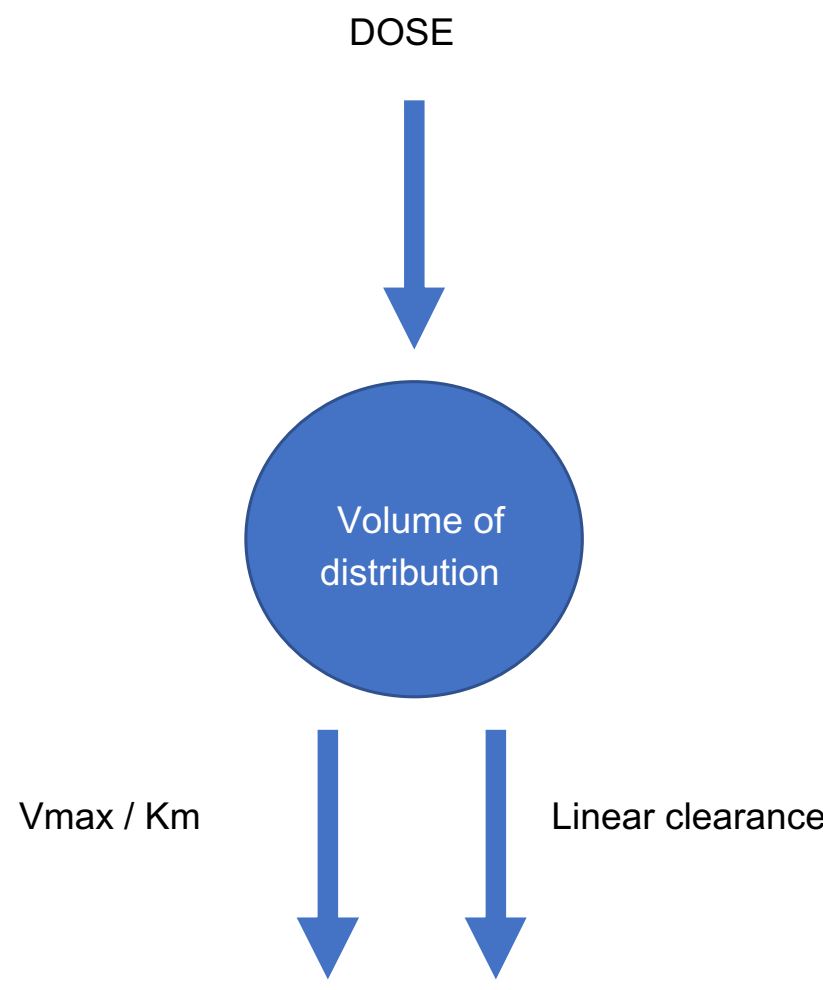

Fig. 1 Schematic representation of the population pharmacokinetic model structure with parallel linear and nonlinear clearance

exposure will be above these thresholds for at least 7 days in all patients. To investigate the potential savings in drug expenses, for the current patient population we calculated the actual drug expenses compared with the drug expenses if everyone switched to a $600 \mathrm{mg}$ fixed dose. The results are presented in ESM Table S3. On average, 20.3\% of drug expenses could be saved.

\subsection{Personalized Dosing Regimens}

When administering tocilizumab to severe COVID-19 patients, one might want to realize a more personalized duration of tocilizumab exposure, since the duration of the hyperinflammation is highly variable between patients. Instead of giving a high dose once, multiple lower doses might be more suitable to personalize the tocilizumab treatment depending on the duration of the hyperinflammation period of the individual patient. In theory, the maximum anti-inflammatory effect of tocilizumab will be reached once the receptor saturation is complete, since exposure above this threshold ( 1 or $5 \mu \mathrm{g} / \mathrm{mL}$ ) will lead to more pronounced saturation of the nonlinear CL. Therefore, different dosing strategies with the same cumulative dose were simulated and are presented in Fig. 9 and ESM Fig. S9 (normal scale) together with these thresholds. A single dose of $800 \mathrm{mg}$ was compared with two doses of $400 \mathrm{mg}$ with an interval of 10 days and four doses of $200 \mathrm{mg}$ with an interval of 7 days. Repeated dosing with lower doses (and lower cumulative doses) results in longer durations of tocilizumab exposure above the saturation thresholds compared with a single high dose of $800 \mathrm{mg}$.

\section{Discussion}

This is the first population pharmacokinetic and descriptive pharmacodynamic study of tocilizumab in severe COVID19 patients admitted to the ICU. The local treatment protocol, which included dexamethasone co-treatment for a maximum of 10 days, was based on the protocol used in the recently published REMAP-CAP trial [4] and national guidelines [10]. Our study shows that tocilizumab pharmacokinetics were variable in the investigated population and were not dependent on bodyweight or other demographic and laboratory covariates. Previous studies have shown that patients with overweight are at risk for a more severe course of COVID-19. Furthermore, in our population, patients with overweight were overrepresented. Dosing tocilizumab based on bodyweight will lead to unnecessarily high tocilizumab concentrations in these patients. Most importantly, our findings indicate that fixed dosing of tocilizumab will decrease IIV exposure compared with the current bodyweight-based dosing, while maintaining efficacy.

The tocilizumab plasma concentration-time courses were best described by a one-compartment pharmacokinetic model with parallel linear and nonlinear elimination, which is characteristic for monoclonal antibodies that target membrane-bound targets that can mediate internalization of a drug-target complex [24]. A two-compartment model that was identified earlier in RA and CRS, with parallel firstorder (linear) and Michaelis-Menten (nonlinear) elimination kinetics, was explored [8, 9, 23]; however, in our dataset, this resulted in an unstable model with extremely high residual standard errors (\%) of the pharmacokinetic parameter estimates, which would require fixation of the $V_{\mathrm{d}}$. Although antibody pharmacokinetics have usually been described using two-compartment models, when the sampling strategy is not intensive on the first day (the distribution phase), the peripheral compartment is not always identifiable [25]. Our findings also show that tocilizumab CL is faster than previously reported for rheumatic diseases $(0.25 \mathrm{~L} /$ day reported by Bastida et al., and $0.29 \mathrm{~L} /$ day reported by Gibiansky and Frey) and in the CAR T-cell-associated CRS (0.5 L/day) $[9,13,15]$. The $V_{\mathrm{d}}$ of $4.34 \mathrm{~L}$ is comparable with that previously reported for rheumatic diseases $(4.83 / \mathrm{L}$ reported by 
Fig. 2 Graphical representation of all individual tocilizumab concentration-time profiles on a semi-log scale (individual predictions, black line) and measured concentrations (blue circles)

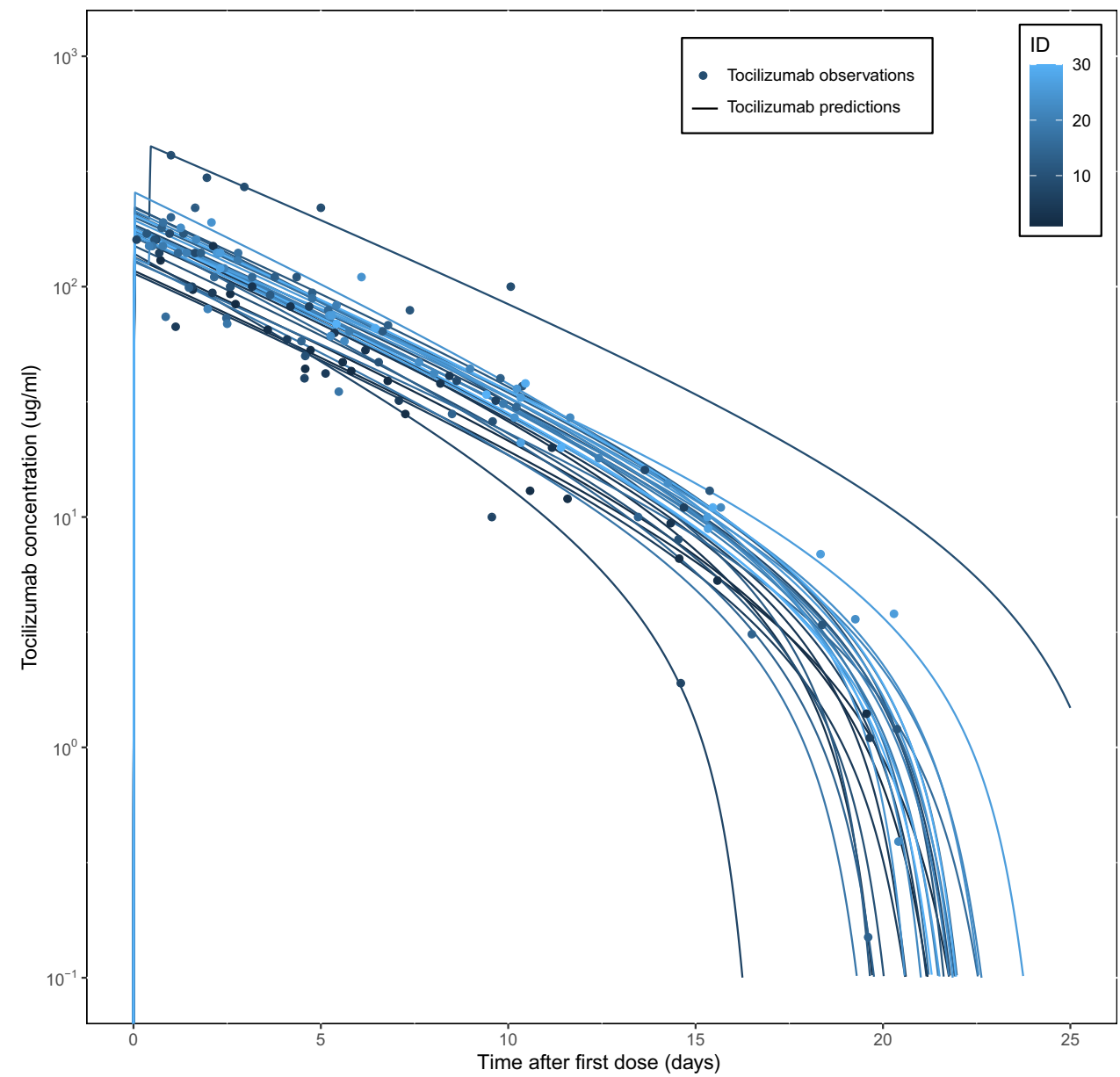

\begin{tabular}{|c|c|c|c|c|c|}
\hline & \multicolumn{3}{|l|}{ Final model } & \multicolumn{2}{|c|}{1000 Bootstrap runs results } \\
\hline & Mean value & RSE (\%) & Shrinkage $(\%)$ & Median value & $95 \% \mathrm{CI}$ \\
\hline CL (L/day) & 0.725 & 4 & & 0.72 & $0.64-0.80$ \\
\hline$V_{\mathrm{d}}(\mathrm{L})$ & 4.34 & 4 & & 4.37 & $3.97-4.78$ \\
\hline$V_{\max }(\mathrm{mg} /$ day $)$ & 4.19 & 17 & & 4.49 & $2.88-8.24$ \\
\hline$K_{\mathrm{m}}(\mathrm{mg} / \mathrm{L})$ & 0.22 & 19 & & 0.30 & $0.02-3.17$ \\
\hline \multicolumn{6}{|c|}{ Interindividual variability } \\
\hline CL (CV\%) & 18.9 & 20 & 8 & 18.2 & $9.5-26.3$ \\
\hline$V_{\mathrm{d}}(\mathrm{CV} \%)$ & 21 & 20 & 11 & 19.4 & $10.0-28.3$ \\
\hline \multicolumn{6}{|c|}{ Random residual variability } \\
\hline Proportional (CV\%) & 17.1 & 8 & 14 & 17.2 & $13.1-20.3$ \\
\hline Additive $(\mu \mathrm{g} / \mathrm{mL})$ & 0.139 & 12 & 14 & 0.1 & $0.0-0.8$ \\
\hline
\end{tabular}

$R S E$ relative standard error, $C I$ confidence interval, $C L$ clearance, $V_{d}$ volume of distribution, $V_{\max }$ maximum elimination rate, $K_{m}$ concentration at which the elimination pathway is half saturated, $C V \%$ percentage coefficient of variation
Bastida et al.; $3.71 V_{1}$ and $3.45 V_{2}$ L/day reported by Gibiansky and Frey $[9,13,15]$. However, the $V_{\max }$ of $4.19 \mathrm{mg} /$ day is slightly different to the $0.239 \mathrm{mg} / \mathrm{h}$ reported by Bastida et al. $(5.76 \mathrm{mg} /$ day), $1.41 \mathrm{mg} / \mathrm{L} /$ day reported by Gibiansky and Frey, and $3.4 \mathrm{mg} /$ day used for the CRS study $[9,13,15]$.
Furthermore, the $K_{\mathrm{m}}$ of $0.22 \mathrm{mg} / \mathrm{L}$ is quite different from the $4.22 \mathrm{mg} / \mathrm{L}$ reported by Bastida et al., and approximately half lower compared with the $0.367 \mathrm{mg} / \mathrm{L}$ reported by Gibiansky and Frey. These differences might be explained by different IL-6-mediated disease activity, different populations, and 
Fig. 3 Graphical representation on a semi-log scale of tocilizumab concentration-time profiles (individual predictions, black line), measured concentrations (blue circles) and CRP pharmacodynamics (red circles), with LOESS regression and 95\% CI (dashed red line with shaded area) of all patients. The $1 \mu \mathrm{g} / \mathrm{mL}$ threshold of complete receptor saturation established in RA patients is presented with a black dashed line. $C R P$ cytokine reactive protein, LOESS locally estimated scatterplot smoothing, $C I$ confidence interval, $R A$ rheumatoid arthritis

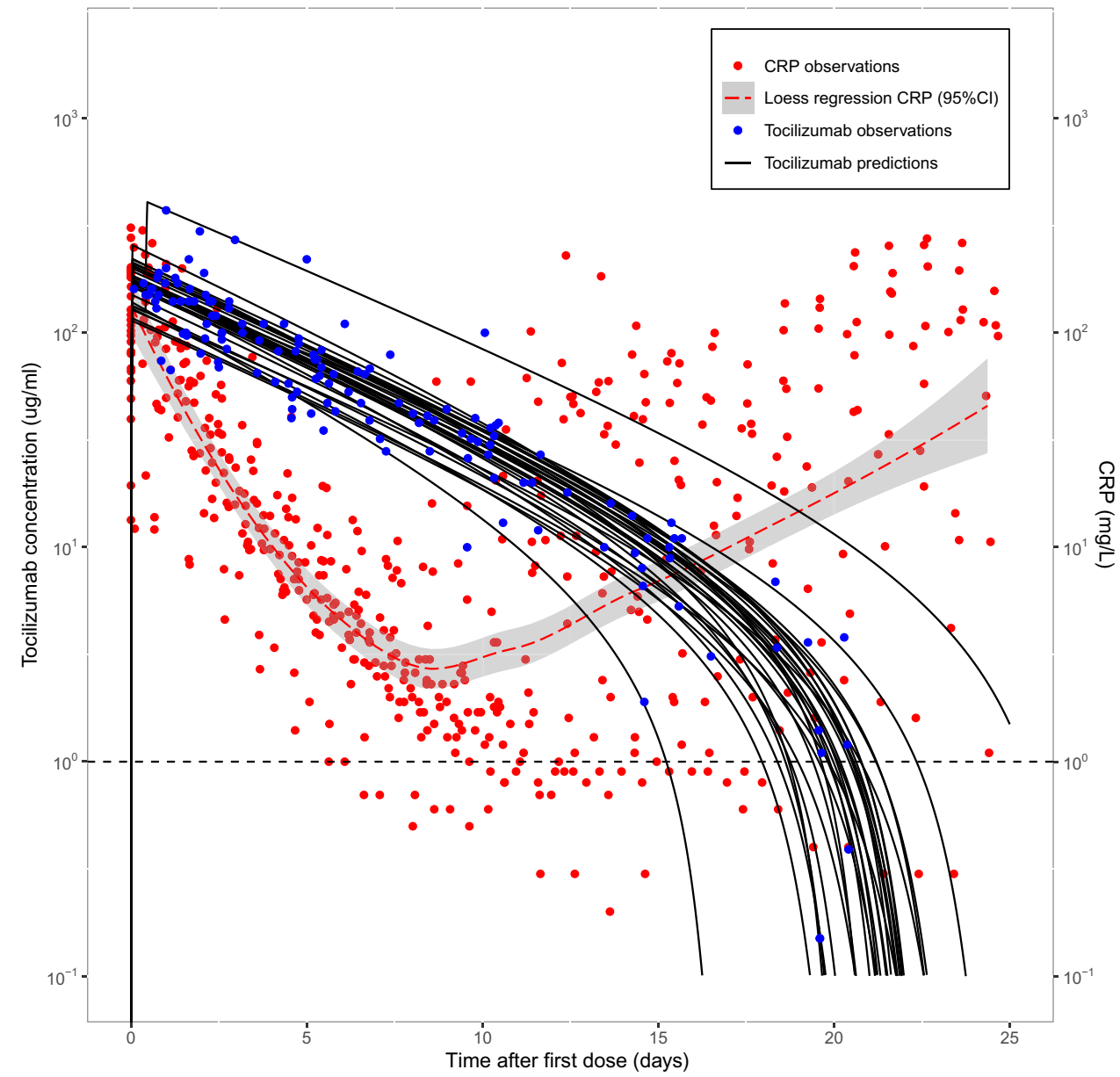

different comedications used in COVID-19 patients, as well as the amount of data available compared with the other study populations.

The descriptive pharmacodynamic results are in line with the work of Nishimoto et al.; CRP dropped rapidly after tocilizumab administration [6]. The elevations of sIL-6R after tocilizumab administration that we observed in our patient population are in line with that observed in healthy volunteers and RA patients [6]. The increase is plasma sIL-6R is probably due to the formation of sIL-6R immune complex, since immune complex formation of antigen and antibody prolongs the half-life of antigen in plasma [26]. The pharmacodynamic results show that a single dose of tocilizumab is sufficient to cover at least the first 10 days after tocilizumab administration. The findings that in some patients a recurrence in the increased CRP levels was observed could be caused by multiple factors, i.e. extended duration of the cytokine storm and/or secondary infections. More research is required to investigate whether additional doses of tocilizumab can be helpful in the subgroup with extended duration of the cytokine storm.

The covariate analysis and simulation results confirm that a fixed-dosing approach for tocilizumab in severe COVID-19 patients reduces variability in exposure among weight categories compared with the current weight-based dosing approach. Dosing based on bodyweight will result in unnecessary high peak concentrations and more variability in time above the saturation threshold between patients. Wang et al. claimed that the best dosing approach for monoclonal antibodies is dependent on the steepness of the relationship between CL and weight [24]. Thus, taking into account that their relationship is generally expressed as a power function covariate model, for exponent values below 0.5 , fixed dosing provides less variability in exposure. In our study, this exponent was estimated to be 0.002 , which is far below the 0.5 above which bodyweight dosing would be rational. This is in line with the findings of Bastida et al., who found a bodyweight exponent of 0.36 , and reconfirms our hypothesis that fixed dosing is a better dosing strategy to achieve a more consistent exposure among all weight groups [15]. Furthermore, we performed posterior power calculations to determine the power ( $95 \%$ confidence) as to whether our study was able to detect a relationship between bodyweight and CL, defined as a bodyweight exponent of 0.5 and 0.8 , respectively. We identified a power of $82 \%$ for identifying a bodyweight exponent of 0.5 , and $99 \%$ power for identifying 

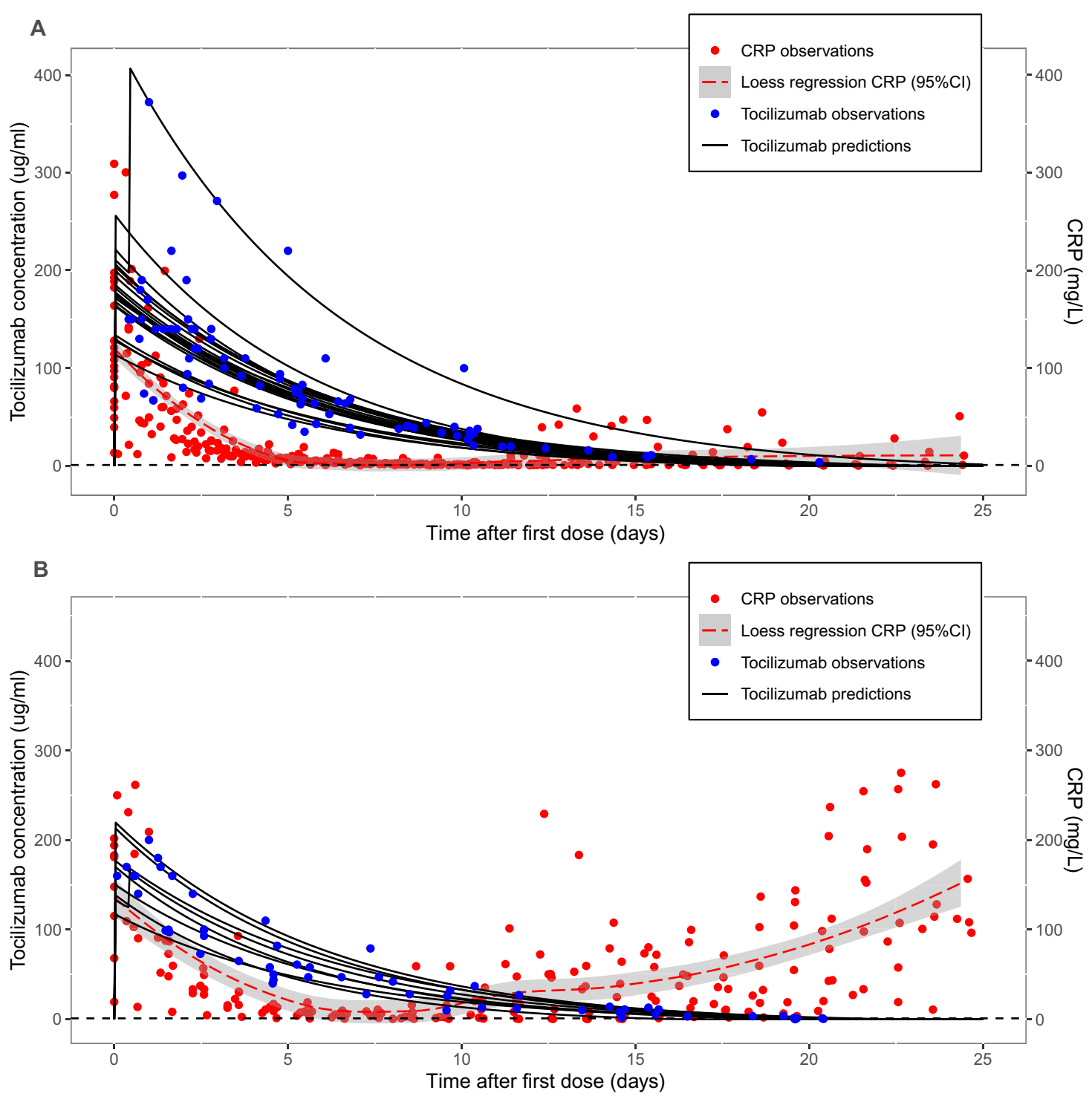

Fig. 4 Graphical representation on a normal scale of tocilizumab concentration-time profiles (individual predictions, black line), measured concentrations (blue circles) and CRP pharmacodynamics (red circles) with LOESS regression and 95\% CI (dashed red line with shaded area) of the patients A without a clear CRP increase relapse

a bodyweight exponent of 0.8 . This indicates that our dataset was powered to detect an exponent that would justify bodyweight dosing (exponents $>0.5$ ). The absence of clear relationships with the other demographic and laboratory covariates are in line with previous findings in RA patients when taking into account the specific population characteristics of the current cohort $[8,15]$.

In patients with RA and Castleman's disease, Nishimoto et al. showed that as long as free tocilizumab was detectable, meaning that at a concentration of $1 \mu \mathrm{g} / \mathrm{mL}$, sIL-6R was saturated with tocilizumab and that IL-6 signalling was completely inhibited [6]. Our own population $(n=21)$, and B with a clear CRP increase relapse $(n=8)$. The $1 \mu \mathrm{g} /$ $\mathrm{mL}$ threshold of complete receptor saturation established in RA patients is presented with a black dashed line. $C R P$ cytokine reactive protein, LOESS locally estimated scatterplot smoothing, CI confidence interval, $R A$ rheumatoid arthritis

pharmacokinetic-based analysis of the ratio between linear and nonlinear CL across the observed tocilizumab concentration range suggests a saturation threshold of $5 \mu \mathrm{g} /$ $\mathrm{mL}$, which is close to the $1 \mu \mathrm{g} / \mathrm{mL}$ threshold. This population pharmacokinetic-based threshold analysis has also been applied previously for the IL-6 receptor blocking agent sarilumab to confirm the most optimal dosing interval [27]. Whether these findings can be extrapolated to all used treatment protocols of COVID-19 patients warrants further investigation. This would indicate that even doses below $600 \mathrm{mg}$ are as effective as the current $8 \mathrm{mg} / \mathrm{kg}$ dose. This hypothesis is supported by findings that a fixed dose 
Fig. 5 Graphical representation on a semi-log scale of tocilizumab concentration-time profiles (individual predictions, black line), measured concentrations (blue circles) and sIL-6r pharmacodynamics (green circles) with LOESS regression and 95\% CI (dashed green line with shaded area) of all patients. The $1 \mu \mathrm{g} / \mathrm{mL}$ threshold of complete receptor saturation established in RA patients is presented with a black dashed line. $s I L-6 r$ soluble interleukin-6 receptor, LOESS locally estimated scatterplot smoothing, $C I$ confidence interval, $R A$ rheumatoid arthritis

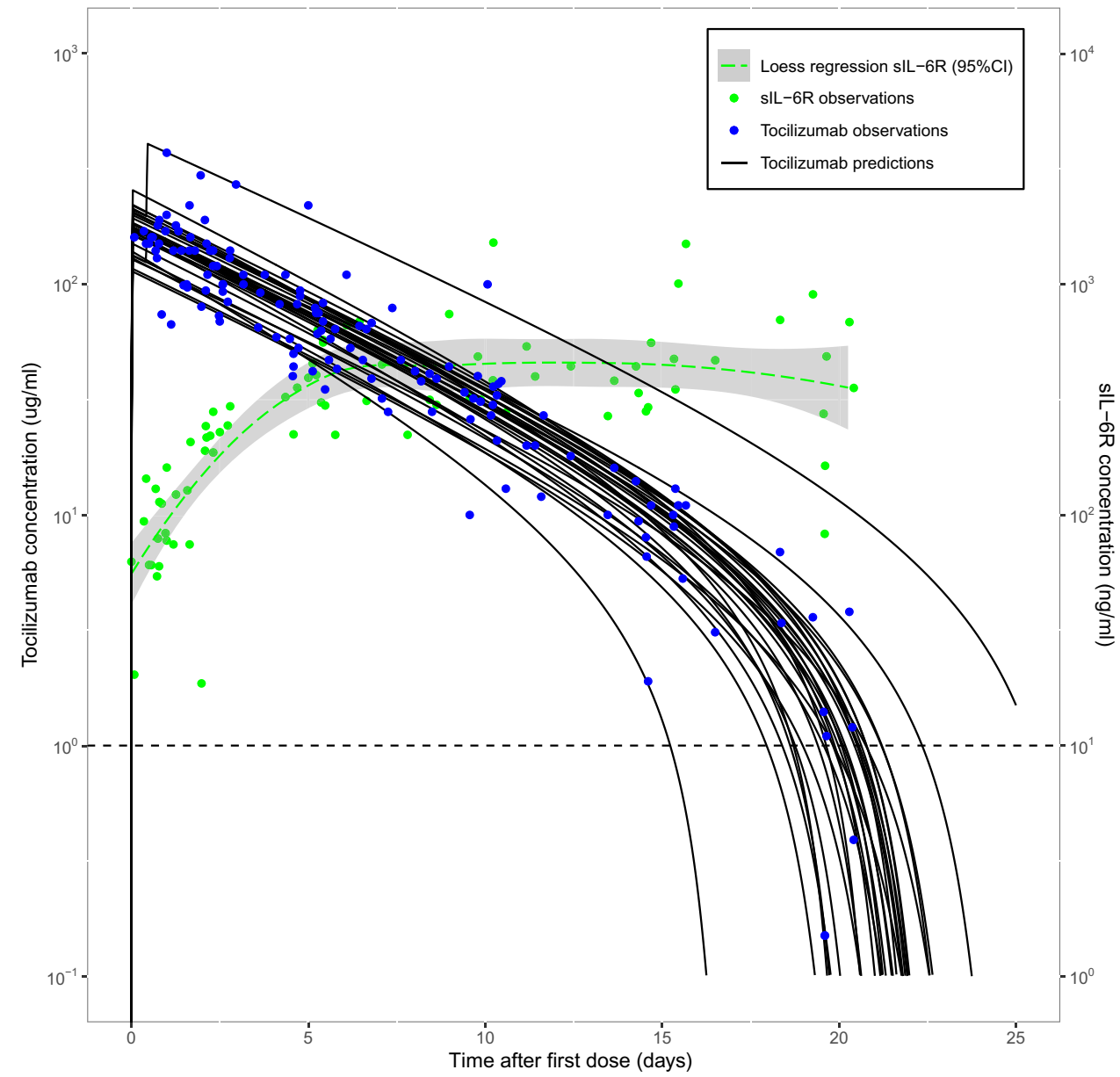

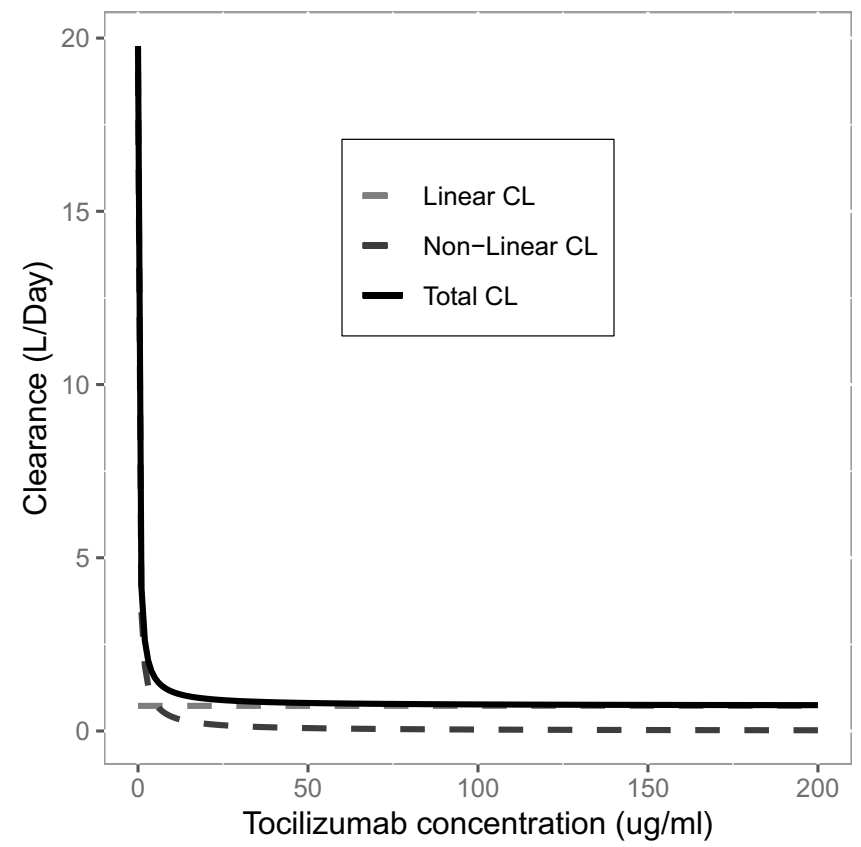

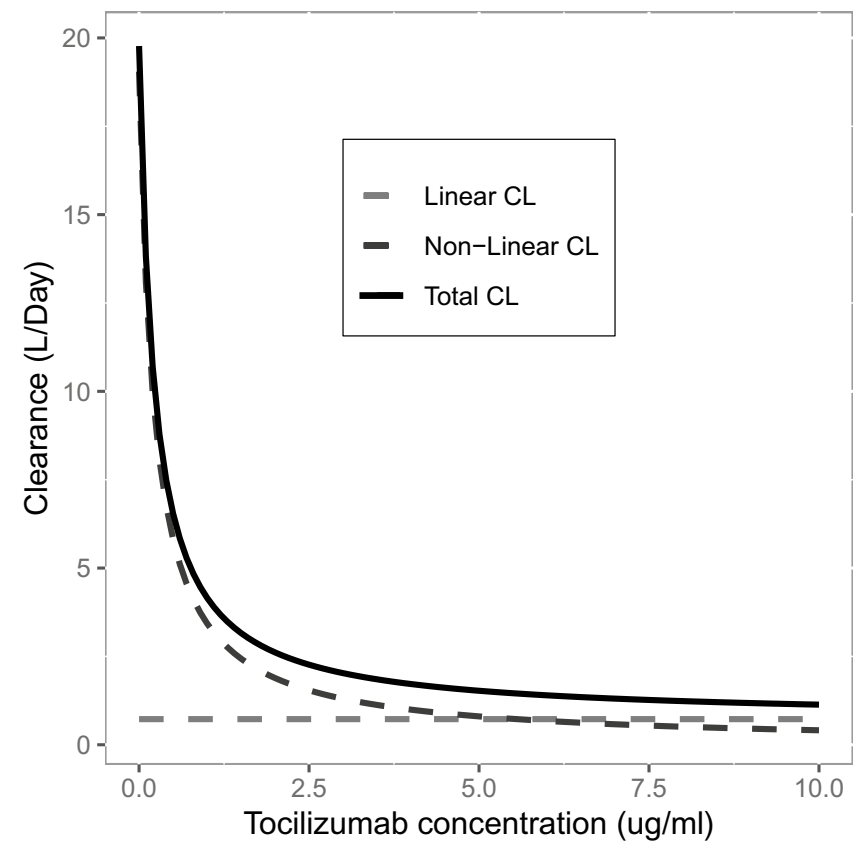

Fig. 6 Ratio between linear clearance, nonlinear clearance (target-mediated drug disposition) and total clearance of the total tocilizumab concentration range derived from the final population pharmacokinetic model parameters 

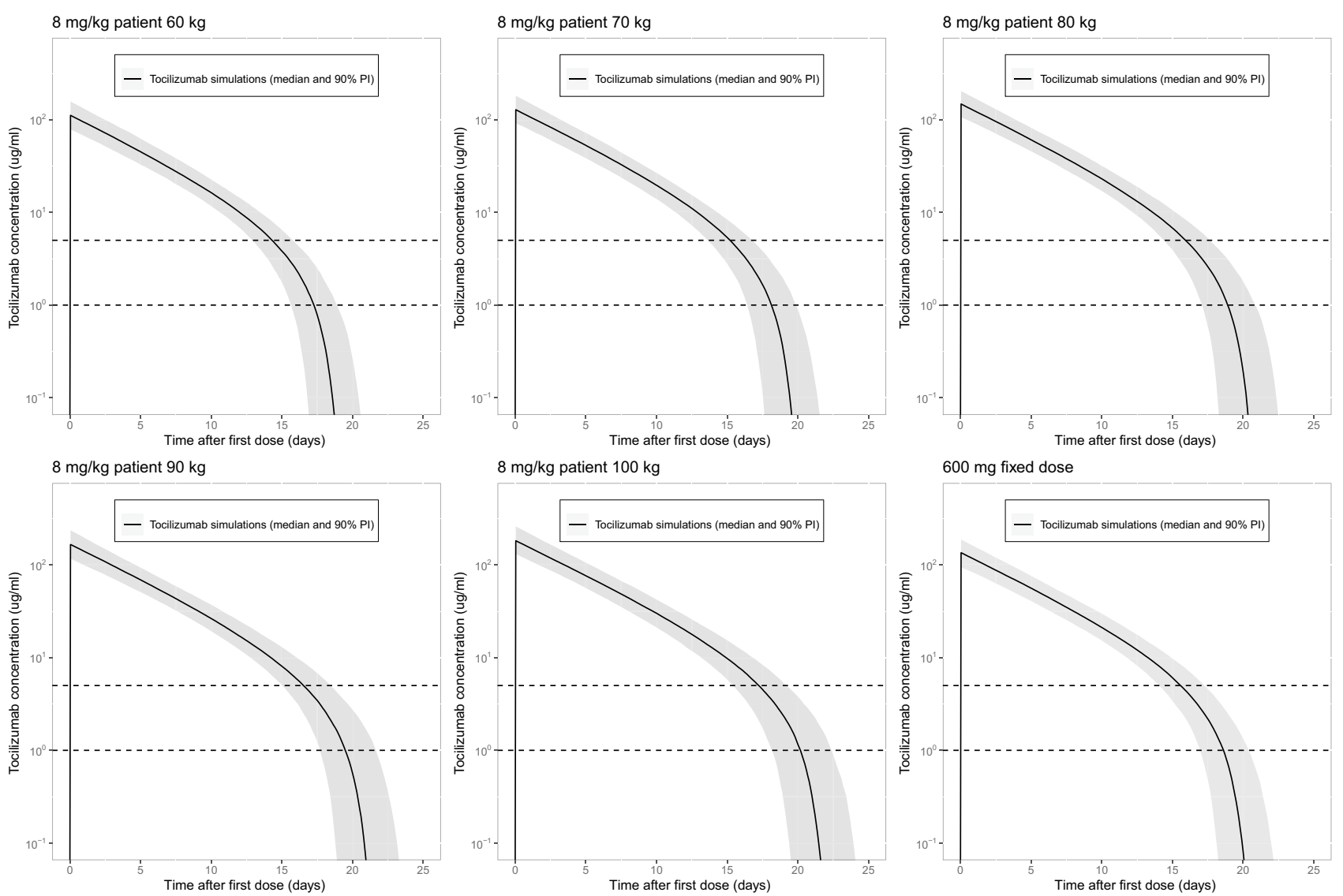

Fig. 7 Variability in tocilizumab exposure when simulating 1000 patients of $60,70,80,90$ and $100 \mathrm{~kg}$ bodyweight receiving $8 \mathrm{mg} /$ $\mathrm{kg}$ dosing versus $600 \mathrm{mg}$ fixed dosing of tocilizumab on a semi-log scale. Median is presented with a solid black line and $90 \%$ prediction

of intravenous tocilizumab $400 \mathrm{mg}$ also leads to rapid CRP decline and improved survival compared with controls who were not treated [28]. Furthermore, a retrospective cohort study showed that subcutaneous administration of tocilizumab $324 \mathrm{mg}$ showed comparable survival as an $8 \mathrm{mg} / \mathrm{kg}$ intravenous administration [29]. Nevertheless, new protocols with fixed doses lower than $600 \mathrm{mg}$, especially in the ICU population, should be carefully investigated and validated before implementation. The timing of tocilizumab administration seems to be the most important factor for success, as shown in the REMAP-CAP trial, and the dose can still be optimized [4]. In cases with shorter duration of the hyperinflammation period, doses can be possibly omitted, with the advantage of reducing risk of superinfections and savings on drug expenses.

Since the RECOVERY and REMAP-CAP open-label, randomized trials have demonstrated an effect of tocilizumab on COVID-19-associated mortality, treatment with IL-6 receptor antagonists has now been embedded in national and international treatment guidelines [10, 30]; however, the effects of different dosing schedules have not interval is presented with a gray or red shaded area. The 1 and $5 \mu \mathrm{g} /$ $\mathrm{mL}$ thresholds of complete receptor saturation are presented with a black dashed line

been investigated in these trials. Because obesity is a risk factor for the development of severe COVID-19, concerns have been raised about overtreatment as well as undertreatment through weight-based dosing. Due to the ongoing pandemic, shortages of tocilizumab and other IL-6 receptor antagonists may be anticipated. A fixed-dose regimen has many practical advantages, including a reduction in dosing errors and avoidance of unnecessary wastage of medication. Furthermore, according to the data presented in this study, relative underdosing of patients with low, or low-normal bodyweight compared with patients with high bodyweight will be avoided.

Our study has some limitations. Due to the observational study design and unpredictable course of COVID-19 patients during ICU admission, sampling could be not performed in an equal manner for all patients and the first day of administration could not be sampled intensively. Nevertheless, the population approach used in the analysis allows solid conclusions to be drawn from unevenly distributed datasets. Furthermore, a total of no less than 139 samples resulted in reliable pharmacokinetic parameter estimates. 

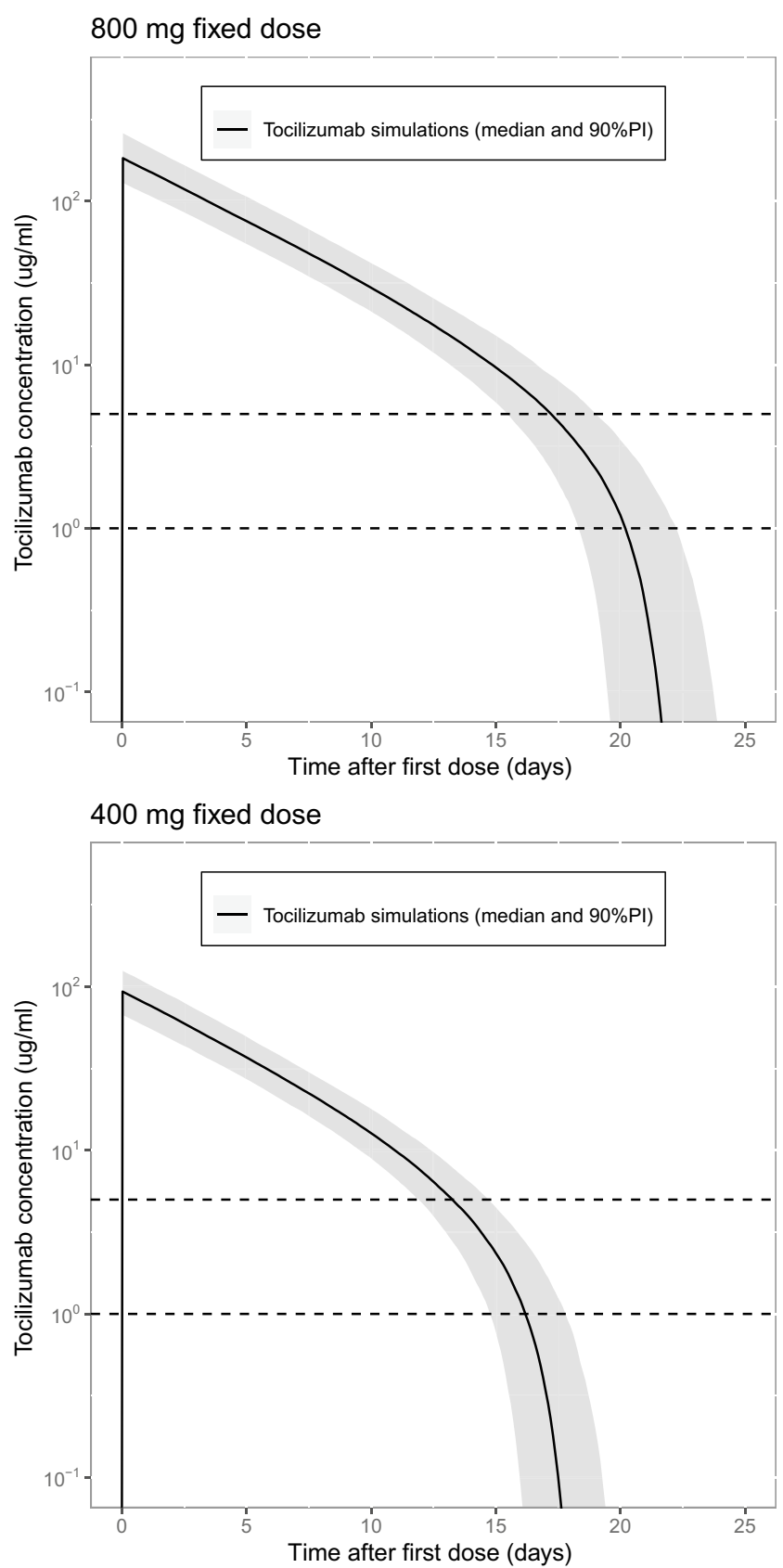

Fig. 8 Variability in exposure when simulating 1000 patients receiving $800,600,400$ or $200 \mathrm{mg}$ fixed dose of tocilizumab on a semi-log scale. Median is presented with a solid black line and $90 \%$ predic-

The observation period was therefore mainly determined by hospital admission time, which might introduce bias for the pharmacodynamic part. Those patients who recovered fast were discharged earlier from the hospital, resulting in a relatively short follow-up time. This results in missing CRP and sIL-6r data after 15 days for patients who recovered fast or died before this moment. Therefore it was not possible to establish a reliable full pharmacokinetic/pharmacodynamic

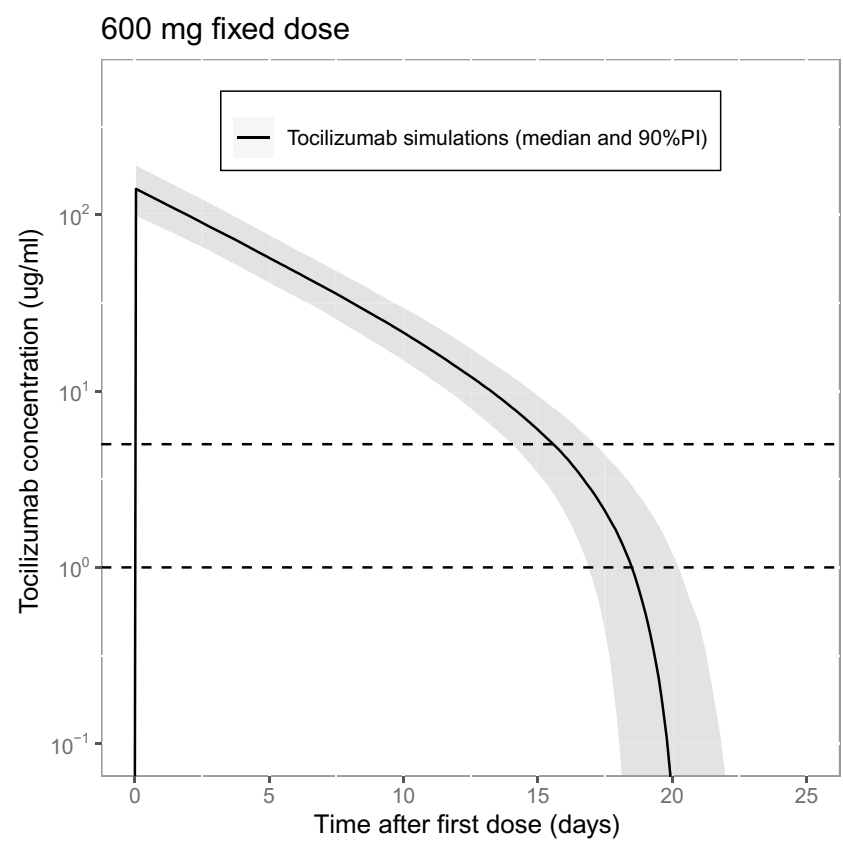

$200 \mathrm{mg}$ fixed dose

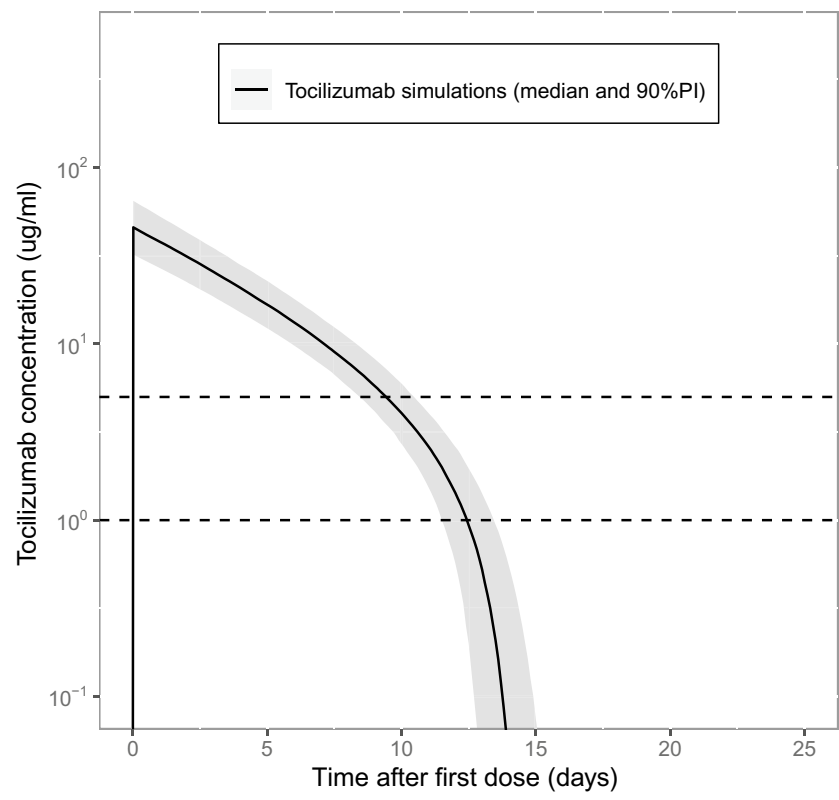

tion interval is presented with a gray shaded area. The 1 and $5 \mu \mathrm{g} / \mathrm{mL}$ thresholds of complete receptor saturation are presented with a black dashed line

model using the current dataset. Ideally, all patients would have the same observation period. The sample size is relatively low but is similar to the dataset that was required (30 patients, 140 samples) to obtain an FDA registration for CRS of tocilizumab, and model performance was good [9]. Furthermore, the posterior power calculation showed that we had a power of at least $82 \%$ to detect a bodyweight exponent of at least 0.5 , which would justify bodyweight-based 

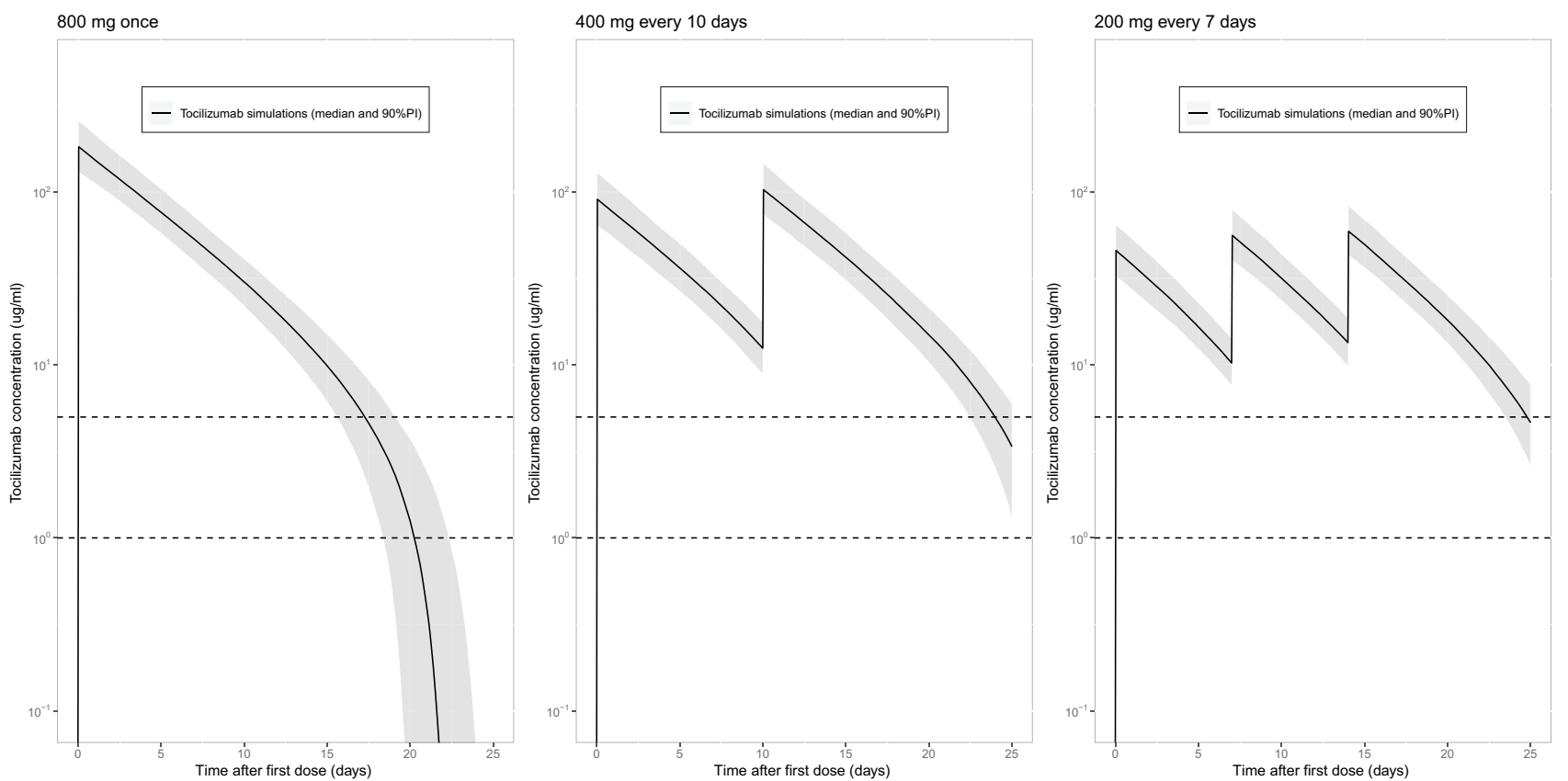

Fig. 9 Simulated exposure of 1000 patients with alternative tocilizumab dosing strategies, $800 \mathrm{mg}$ once, versus $400 \mathrm{mg}$ every 10 days and $200 \mathrm{mg}$ every 7 days on a semi-log scale. Median is presented

dosing. Since we had only a limited number of patients with bodyweights below $75 \mathrm{~kg}$, and no patients with a bodyweight below $55 \mathrm{~kg}$, the findings should not be extrapolated to patients with lower bodyweights, without careful validation. However, since the majority of the patients on the ICU ward have a bodyweight of $\geq 75 \mathrm{~kg}$, our findings will apply for most of the admitted patients.

\section{Conclusions}

Our findings strongly support fixed dosing of tocilizumab in ICU-admitted COVID-19 patients at a dose of $600 \mathrm{mg}$, as it leads to less variability in exposure compared with the currently applied bodyweight dosing. In patients with low bodyweight, the fixed dose will avoid relative underexposure, while in patients with high bodyweight, the fixed dosing strategy will avoid unnecessary overexposure and excessive drug expenses. Further pharmacokinetic and pharmacodynamic research is required to investigate whether alternative cost-saving regimens with even lower doses than $600 \mathrm{mg}$ are as well tolerated and effective.

Supplementary Information The online version contains supplementary material available at https://doi.org/10.1007/s40262-021-01074-2.

Acknowledgements The authors are indebted to all the patients with COVID-19 who participated in this research. They sincerely thank all with a solid black line and $90 \%$ prediction interval is presented with a gray shaded area. The 1 and $5 \mu \mathrm{g} / \mathrm{mL}$ thresholds of complete receptor saturation are presented with a black dashed line

medical students, laboratory staff and clinical staff of the Leiden University Medical Center who, until this time, contributed to obtaining the data. The authors would also like to thank Marjolein van Wolfswinkel (Department of Infectious Diseases) for performing the sIL-6R measurements.

\section{Declarations}

Funding No external funding was received for this work

Conflicts of interest Dirk Jan A. R. Moes, David J. van Westerloo, Sandra M. Arend, Jesse J. Swen, Annick de Vries, Henk-Jan Guchelaar, Simone A. Joosten, Mark G. J. de Boer and Judith van Paasen have no conflicts of interest to declare. Teun van Gelder has received lecture fees and study grants from Chiesi and Astellas, in addition to consulting fees from Roche Diagnostics, Vitaeris, CSL Behring, Astellas, Aurinia Pharma and Novartis.

Ethics approval This study was conducted in compliance with the Declaration of Helsinki and approved by the COVID-19 Scientific and Ethics Committee of the Leiden University Medical Center with number coco 2020-033.

Consent to participate The COVID-19 Scientific and Ethics Committee of the Leiden University Medical Center granted a waiver for the requirement for informed consent.

Availability of data and material The datasets generated and/or analyzed during this study are available from the corresponding author upon reasonable request.

Code availability The NONMEM code used to generate and/or analyse the data described in this study is provided in the ESM. 
Author contributions DJARM, DJvW, SMA, TvG and JvP designed the research protocol. DJARM wrote the initial draft of the manuscript. DJARM, DJvW, SMA, TvG, JvP, JJS, AdV, HJG, SAJ and MGJdB wrote and reviewed the manuscript. DJARM, DJvW, JvP and SAJ performed the research. DJARM, DJvW, SMA, TvG, JvP, JJS, AdV, HJG, SAJ and MGJdB analyzed and checked the data. AdV, SAJ, MGJdB and SMA contributed new reagents/analytical tools.

Open Access This article is licensed under a Creative Commons Attribution-NonCommercial 4.0 International License, which permits any non-commercial use, sharing, adaptation, distribution and reproduction in any medium or format, as long as you give appropriate credit to the original author(s) and the source, provide a link to the Creative Commons licence, and indicate if changes were made. The images or other third party material in this article are included in the article's Creative Commons licence, unless indicated otherwise in a credit line to the material. If material is not included in the article's Creative Commons licence and your intended use is not permitted by statutory regulation or exceeds the permitted use, you will need to obtain permission directly from the copyright holder. To view a copy of this licence, visit http://creativecommons.org/licenses/by-nc/4.0/.

\section{References}

1. Worldometers.info. https://www.worldometers.info/coronavirus/. Accessed 24 Aug 2021.

2. Hu B, Guo H, Zhou P, Shi ZL. Characteristics of SARS-CoV-2 and COVID-19. Nat Rev Microbiol. 2021;19(3):141-54.

3. The WHO Rapid Evidence Appraisal for COVID-19 Therapies (REACT) Working Group, Sterne JAC, Murthy S, Diaz JV, Slutsky AS, Villar J, et al. Association between administration of systemic corticosteroids and mortality among critically ill patients with COVID-19: a meta-analysis. JAMA. 2020;324(13):1330-41.

4. The REMAP-CAP Investigators, Gordon AC, Mouncey PR, AlBeidh F, Rowan KM, Nichol AD, et al. Interleukin-6 receptor antagonists in critically ill patients with Covid-19. N Engl J Med. 2021;384:1491-502.

5. RECOVERY Collaborative Group, Horby P, Lim WS, Emberson JR, Mafham M, Bell JL, et al. Dexamethasone in hospitalized patients with Covid-19. N Engl J Med. 2021;384(8):693-704.

6. Nishimoto N, Terao K, Mima T, Nakahara H, Takagi N, Kakehi $\mathrm{T}$. Mechanisms and pathologic significances in increase in serum interleukin-6 (IL-6) and soluble IL-6 receptor after administration of an anti-IL- 6 receptor antibody, tocilizumab, in patients with rheumatoid arthritis and Castleman disease. Blood. 2008;112(10):3959-64.

7. Tanaka T, Narazaki M, Kishimoto T. Anti-interleukin-6 receptor antibody, tocilizumab, for the treatment of autoimmune diseases. FEBS Lett. 2011;585(23):3699-709.

8. US FDA. Clinical pharmacology and biopharmaceutics review(s) Actemra (tocilizumab). Silver Spring: US FDA; 2008.

9. US FDA. FDA multi-discipline review tocilizumab cytokine release syndrome. Silver Spring: US FDA; 2017.

10. Stichting Werkgroep Antibioticabeleid. Medicamenteuze behandeling voor patiënten met COVID-19 (infectie met SARS-CoV-2). 2021. https://swab.nl/nl/covid-19. Accessed 1 Aug 2021.

11. Kneepkens EL, van den Oever I, Plasencia CH, Pascual-Salcedo D, de Vries A, Hart M, et al. Serum tocilizumab trough concentration can be used to monitor systemic IL-6 receptor blockade in patients with rheumatoid arthritis: a prospective observational cohort study. Scand J Rheumatol. 2017;46(2):87-94.

12. Rispens T, Leeuwen A, Vennegoor A, Killestein J, Aalberse RC, Wolbink GJ, et al. Measurement of serum levels of natalizumab, an immunoglobulin $\mathrm{G} 4$ therapeutic monoclonal antibody. Anal Biochem. 2011;411(2):271-6.

13. Gibiansky L, Frey N. Linking interleukin-6 receptor blockade with tocilizumab and its hematological effects using a modeling approach. J Pharmacokinet Pharmacodyn. 2012;39(1):5-16.

14. Oude Munnink TH, Henstra MJ, Segerink LI, Movig KL, Brummelhuis-Visser P. Therapeutic drug monitoring of monoclonal antibodies in inflammatory and malignant disease: translating TNF-alpha experience to oncology. Clin Pharmacol Ther. 2016;99(4):419-31.

15. Bastida C, Ruiz-Esquide V, Pascal M, de Vries Schultink AHM, Yague J, Sanmarti R, et al. Fixed dosing of intravenous tocilizumab in rheumatoid arthritis. Results from a population pharmacokinetic analysis. Br J Clin Pharmacol. 2018;84(4):716-25.

16. Xiang Z, Liu J, Shi D, Chen W, Li J, Yan R, et al. Glucocorticoids improve severe or critical COVID-19 by activating ACE2 and reducing IL-6 levels. Int J Biol Sci. 2020;16(13):2382-91.

17. Beal S, Sheiner LB, Boeckmann A. NONMEM user's guides (1989-2006). Ellicott: ICON Development Solutions; 2006.

18. Jonsson EN, Karlsson MO. Xpose-an S-PLUS based population pharmacokinetic/pharmacodynamic model building aid for NONMEM. Comput Methods Programs Biomed. 1999;58(1):51-64.

19. Lindbom L, Pihlgren P, Jonsson EN. PsN-Toolkit-a collection of computer intensive statistical methods for non-linear mixed effect modeling using NONMEM. Comput Methods Programs Biomed. 2005;79(3):241-57.

20. Keizer RJ, van Benten M, Beijnen JH, Schellens JH, Huitema AD. Pirana and PCluster: a modeling environment and cluster infrastructure for NONMEM. Comput Methods Programs Biomed. 2011;101(1):72-9.

21. Comets E, Brendel K, Mentre F. Computing normalised prediction distribution errors to evaluate nonlinear mixed-effect models: the npde add-on package for R. Comput Methods Programs Biomed. 2008;90(2):154-66.

22. R Development Core Team. R: a language and environment for statistical computing. Vienna: R Foundation for Statistical Computing; 2013.

23. Levi M, Grange S, Frey N. Exposure-response relationship of tocilizumab, an anti-IL-6 receptor monoclonal antibody, in a large population of patients with rheumatoid arthritis. J Clin Pharmacol. 2013;53(2):151-9.

24. Wang DD, Zhang S, Zhao H, Men AY, Parivar K. Fixed dosing versus body size-based dosing of monoclonal antibodies in adult clinical trials. J Clin Pharmacol. 2009;49(9):1012-24.

25. Passot C, Pouw MF, Mulleman D, Bejan-Angoulvant T, Paintaud $\mathrm{G}$, Dreesen E, et al. Therapeutic drug monitoring of biopharmaceuticals may benefit from pharmacokinetic and pharmacokineticpharmacodynamic modeling. Ther Drug Monit. 2017;39(4):322-6.

26. Montero-Julian FA, Klein B, Gautherot E, Brailly H. Pharmacokinetic study of anti-interleukin-6 (IL-6) therapy with monoclonal antibodies: enhancement of IL- 6 clearance by cocktails of antiIL-6 antibodies. Blood. 1995;85(4):917-24.

27. Xu C, Su Y, Paccaly A, Kanamaluru V. Population pharmacokinetics of sarilumab in patients with rheumatoid arthritis. Clin Pharmacokinet. 2019;58(11):1455-67.

28. De Rossi N, Scarpazza C, Filippini C, Cordioli C, Rasia S, Mancinelli CR, et al. Early use of low dose tocilizumab in patients with COVID-19: A retrospective cohort study with a complete follow-up. EClinicalMedicine. 2020;25:100459.

29. Guaraldi G, Meschiari M, Cozzi-Lepri A, Milic J, Tonelli $\mathrm{R}$, Menozzi M, et al. Tocilizumab in patients with severe COVID-19: a retrospective cohort study. Lancet Rheumatol. 2020;2(8):e474-84.

30. World Health Organization. COVID-19 Clinical management: living guidance. 2021. Report No.: WHO/2019-nCoV/clinical/2021.1. https://www.who.int/publications/i/item/WHO-2019nCoV-clinical-2021-1. Accessed 1 Aug 2021. 


\section{Authors and Affiliations}

Dirk Jan A. R. Moes ${ }^{1,2}$ (1) - David J. van Westerloo ${ }^{3}$. Sandra M. Arend ${ }^{4}$. Jesse J. Swen ${ }^{1,2} \cdot$ Annick de Vries $^{5}$. Henk-Jan Guchelaar ${ }^{1,2} \cdot$ Simone A. Joosten ${ }^{4}$. Mark G. J. de Boer ${ }^{4} \cdot$ Teun van Gelder $^{1,2}$. Judith van Paassen ${ }^{3}$

$\triangle$ Dirk Jan A. R. Moes

D.J.A.R.Moes@lumc.nl

1 Department of Clinical Pharmacy and Toxicology, Leiden University Medical Center, Albinusdreef 2, 2333 ZA Leiden, The Netherlands

2 Leiden Network for Personalised Therapeutics, Albinusdreef 2, 2333 ZA Leiden, The Netherlands
3 Department of Intensive Care, Leiden University Medical Center, Albinusdreef 2, 2333 ZA Leiden, The Netherlands

4 Department of Infectious Diseases, Leiden University Medical Center, Albinusdreef 2, 2333 ZA Leiden, The Netherlands

5 Biologics Lab, Sanquin Diagnostic Services, Plesmanlaan 125, 1066 CX Amsterdam, The Netherlands 